


\section{DIE RELIEFS DER TRAIANSSÄULE}


Der geplante erste Text-Band dieses Werkes wird nicht erscheinen. Infolge dessen sind die beiden vorliegenden Textbände umbeziffert worden, statt Bd. II jetzt Bd. I und statt Bd. III jetzt Bd. II. Das Werk ist damit abgeschlossen, es besteht aus Tafelband I und II und Textband I und II. Die Tafelbände enthalten die Reliefs in Handkupferdruck, die Textbände den Kommentar zu den Reliefs. 


\section{DIE RELIEFS \\ DER TRAIANSSÄULE.}

Herausgegeben und historisch erklärt

von

CONRAD CICHORIUS.

Erster Textband:

Commentar zu den Reliefs des ersten dakischen Krieges.

Berlin und Leipzig 1927

Verlag von Walter de Gruyter \& Co. 


\section{DIE}

\section{RELIEFS DER TRAIANSSÄULE.}

Herausgegeben und historisch erklärt

von

\section{CONRAD CICHORIUS.}

Zweiter Textband:

Commentar zu den Reliefs des ersten dakischen Krieges.

Berlin

Verlag von Georg Reimer

1896. 



\title{
THEODOR MOMMSEN
}

\author{
gewidmet.
}


